\title{
Localisation of tumour deposits by external scanning after injection of radiolabelled anti-carcinoembryonic antigen
}

\author{
P W DYKES, K R HINE, A R BRADWELL, J C BLACKBURN, T A REEDER, Z DROLC, \\ $S$ N BOOTH
}

\section{Summary and conclusions}

Sheep IgG antibody to carcinoembryonic antigen (CEA) was radiolabelled with ${ }^{131} I$ and used to identify human gastrointestinal tumours by external subtraction imaging. ${ }^{99 \mathrm{~m} T c-p e r t e c h n e t a t e}$ and ${ }^{99 \mathrm{~m} T c-h u m a n}$ serum albumin were used to identify tissue spaces. In 13 patients with tumours four out of five primary sites and eight out of 11 secondary sites were successfully shown. Two patients with benign disease had negative scans. Comparison with conventional methods of scanning showed good correlation.

The success of this pilot study should encourage the search for more tumour-specific antigens and further study of the implications for treatment.

\section{Introduction}

The possibility that antitumour antibodies might successfully localise to both primary and secondary tumour deposits has attracted a great deal of interest but, until recently, little success, largely because of the absence of tumour-specific antigens. ${ }^{12}$

Carcinoembryonic antigen (CEA) is not tumour specific, but its concentrations are increased in most gastrointestinal neoplasms and in various other cancers, ${ }^{3}$ with high concentrations demonstrable mainly on the tumour-cell surface. ${ }^{45}$

In animal studies both external scanning and direct examination of the tissues showed that there was uptake of radiolabelled antibody to CEA into transplanted tumour deposits. ${ }^{6-9}$ An initial attempt to identify human neoplasms with anti-CEA was unsuccessful, ${ }^{10}$ but recently Goldenberg et al have described 18 patients with various cancers in whom all but six out of 38 tumour deposits were successfully shown using a subtraction technique. ${ }^{11}$ Eleven primary neoplasms were included, together with their metastases, and the only patient in whom no tumour was demonstrable despite the existence of primary and secondary deposits had a lymphocytic lymphoma (which is usually devoid of CEA).

We attempted to localise primary and secondary deposits of various gastrointestinal cancers using a radiolabelled antibody to CEA and to compare the gammacamera images thus obtained with conventional methods of tumour identification.

\footnotetext{
Department of Immunology, University of Birmingham, Birmingham B15 2TJ

K R HINE, BSC, MRCP, clinical research fellow

A R BRADWELL, MB, MRCP, director, immunodiagnostic research laboratory

J C BLACKBURN, technician

S N BOOTH, MD, MRCP, honorary research fellow (now consultant physician, Kidderminster General Hospital)

General Hospital, Birmingham

P W DYKES, MD, FRCP, consultant physician

Department of Nuclear Medicine, Queen Elizabeth Hospital, Birmingham

T A REEDER, BENG, MSC, physicist

Z DROLC, BSC, principal physicist
}

\section{Patients and methods}

ANTIBODY

Antibodies to CEA were produced in sheep by immunisation with CEA purified from hepatic metastases of colonic carcinoma. Crossreaction with other tissue antigens was removed by adsorption with normal liver, colon, lung, and spleen. The antiserum was considered to be satisfactory when there was uptake only on colon tumour-cell surfaces using the immunoperoxidase technique for staining tissue sections. The serum was then fractionated on a DEAE 52 cellulose column and the IgG fraction was affinity-purified using CEA linked to Sepharose 4B. At this stage each batch of antibody was tested for acute toxicity and pyrogenicity in rabbits. All tests were satisfactory. The purified antibody was then labelled under sterile conditions with ${ }^{131}$ I using the chloramine- $T$ method. Free iodide was removed on a Dowex column and the antibody was diluted in $1 \%$ albumin-in-saline solution. The antibody solution was centrifuged for 12 hours at $20000 \mathrm{~g}$ before injection into the patients through a $0.22 \mu \mathrm{m}$ filter.

After the patients had been tested for anaphylactic hypersensitivity the radiolabelled antibody was injected intravenously in 10-15 ml of $1 \%$ albumin-in-saline solution over about 10 minutes. Between $100 \mu \mathrm{g}$ and $200 \mu \mathrm{g}$ of sheep IgG was given, equivalent to $300-1000 \mu \mathrm{Ci}$ of ${ }^{131} \mathrm{I}$ (usual dose: $600-700 \mu \mathrm{Ci}$ ). Only one dose of antibody was given, but before each scan each patient also received intravenous ${ }^{99 \mathrm{~m}} \mathrm{Tc}$-pertechnetate $(200 \mu \mathrm{Ci})$ and ${ }^{99 \mathrm{~m}} \mathrm{Tc}$-labelled human serum albumin $(500 \mu \mathrm{Ci})$, which distribute similarly to free iodide and radiolabelled antibody respectively in the blood pool.

IMAGING

Immediate images of the chest and abdomen were obtained with a gammacamera (Searle LFOV with medium energy collimator) and further scans were taken 4,24 , and 48 hours after the injection of antibody.

The camera was linked to DEC PDP 11/40 computer with a dual isotope facility and a grey-scale visual display unit. The data were stored and displayed in a $64 \times 64$ matrix. Subtraction of the technetium components from the iodine components was performed, after normalising over the cardiac area, to highlight the areas of selective uptake of antibody by CEA-producing tissue.

\section{PATIENTS}

Fifteen patients were studied, in eight of whom large-bowel carcinomas had been removed. In seven of the eight the presence of liver metastases had been confirmed by isotope scanning and in the other patient there was clinical evidence of pelvic recurrence. Three patients had unresected primary rectal tumours; in one, laparoscopy had shown extensive peritoneal deposits, in the second the primary tumour was very advanced locally but there were no demonstrable distant metastases, and in the third the neoplasm proved to be completely resectable at laparotomy seven days after the antibody scans. One patient had an unresected pancreatic primary tumour and at laparotomy had been shown to have secondary deposits in the left lobe of the liver. A further patient underwent laparotomy a few hours after the injection of antibody and was found to have widely disseminated adenocarcinoma, but the primary tumour was not identified. The remaining two patients had large-bowel strictures on barium enema, which were thought likely to be neoplastic. At operation, however, the strictures were found to be benign-in one case in association with diverticular disease and in the other chronic ulcerative colitis.

Thyroid uptake of radioiodine was blocked by the administration of oral potassium iodide, $180 \mathrm{mg} /$ day, starting at least 24 hours before the injection of antibody and continuing for three weeks. Three patients 
received affinity-purified antibody; in the remainder this step in the preparation was omitted. In 14 patients, regular blood samples were taken during the first five hours and again at 24 hours. Six patients collected 24-hour urine samples to assess the excretion of free iodide. All patients gave their informed consent to the procedure.

\section{Results}

Four of the five patients with primary tumours had them demonstrated on the antibody scans. The one exception was the patient (case 13) with disseminated adenocarcinoma from an undetermined primary tumour in whom only one scan (at four hours) was performed (see table). Local extension of the primary tumour to the right pelvic wall was shown in case 10 (fig 1), which was obvious on clinical examination and suggested by computed tomography (fig 2). Eleven

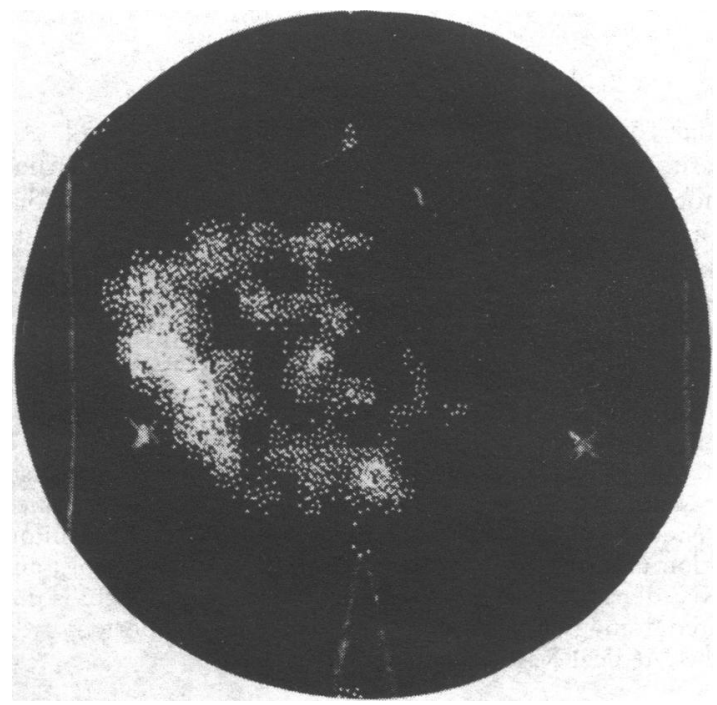

FIG 1-Case 10. Abdominal view of antibody image showing local extension of primary rectosigmoid carcinoma on to right pelvic wall. Crosses indicate anterior, superior iliac spines.

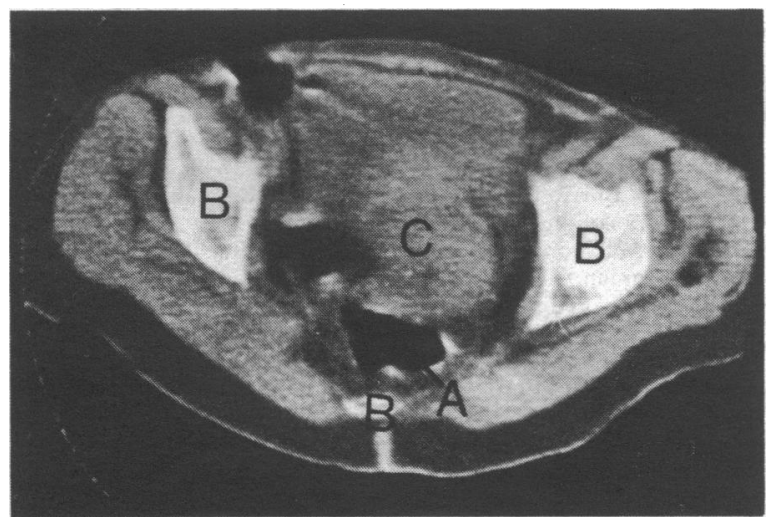

FIG 2-Case 10. Pelvic view of computed tomogram showing large primary carcinoma of rectosigmoid junction.

$A=$ rectum,$B=$ pelvic bones, $C=$ carcinoma

patients were known to have remote metastases and these were successfully identified in eight. The three exceptions included case 13 and a patient (case 3) with a large solitary liver metastasis $(25 \times 20 \mathrm{~cm})$ which both iodine and technetium failed to penetrate. In the two patients (cases 14 and 15) who were subsequently shown at laparotomy to have benign colonic strictures all scans were negative.

The distribution of demonstrable tumour on the antibody scans correlated well with conventional methods (figs 1-4), and positive scans were obtained in five out of six patients in whom metastases were shown at laparotomy.

The serum CEA concentration bore no obvious relation to the chances of achieving a positive scan and values of up to $16000 \mathrm{mg} / 1 \mathrm{did}$ not prevent the demonstration of hepatic metastases (case 2; figs 3 and 4). In 14 patients we studied the serial changes in serum CEA after injection of the antibody. Three patients showed changes of less than $10 \%$ (assay variation), five showed an immediate fall of less than $25 \%$, and the remaining six showed falls of $25-50 \%$. The serum CEA values returned to preinjection levels within 24 hours. The pattern of CEA change did not appear to correlate with either the absolute preinjection value of CEA or the chances of achieving a positive scan.

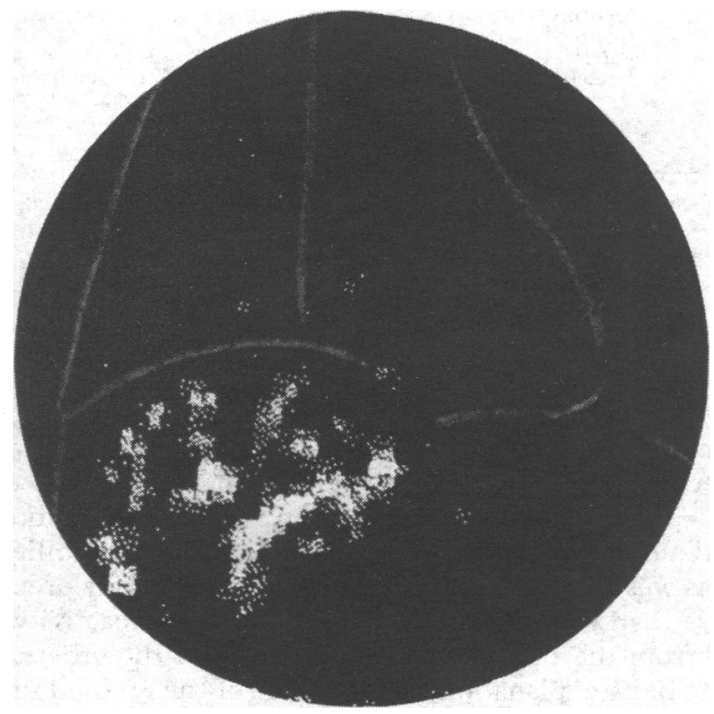

FIG 3-Case 2. Chest view of antibody image showing areas of increased uptake of iodine-labelled antibody in liver. Heart and lungs are fully subtracted and their position is indicated.

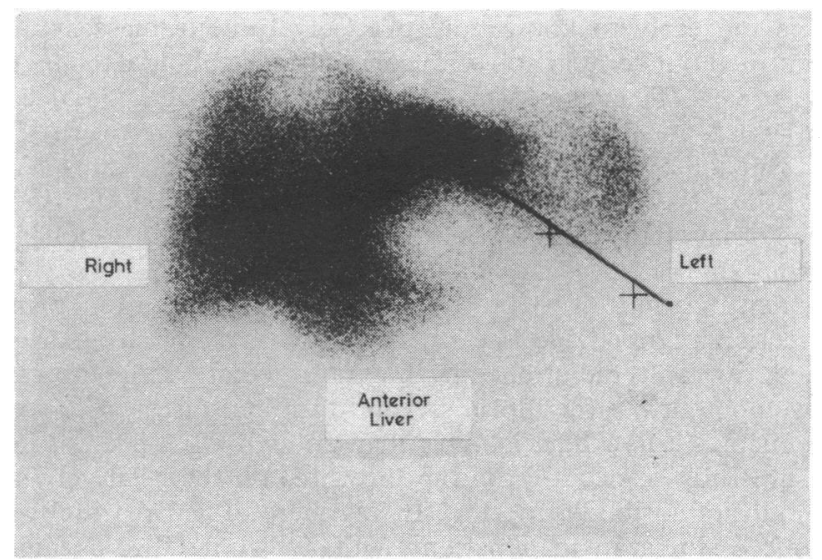

FIG 4-Case 2. Colloidal technetium liver scan showing areas of decreased uptake consistent with metastases.

Urine collections for free iodide in six patients showed that $4 \%$ to $14 \%$ of the total iodine administered was excreted in the first 24 hours (see table). This appeared to bear no relation to either the serum CEA concentration or the pattern of change in serum CEA after injection of the antibody.

Fever $\left(38.1^{\circ} \mathrm{C}\right)$ was observed in only one patient; it was maximal eight hours after injection and disappeared nine hours later. The fever was unaccompanied by any subjective change. Hourly temperature and pulse recordings were normal in all other patients.

\section{Discussion}

We have confirmed that antibody scanning can show tumour deposits, and the visualisation of both primary and secondary lesions was remarkably clear. In three patients, however, the technique was totally unsuccessful. Of these, one patient had 
Summary of results in 15 patients scanned for the presence of gastrointestinal carcinomas

\begin{tabular}{|c|c|c|c|c|c|c|c|c|c|c|}
\hline \multirow{2}{*}{$\begin{array}{l}\text { Case } \\
\text { No }\end{array}$} & \multicolumn{2}{|c|}{ Primary tumour } & \multicolumn{2}{|c|}{ Secondary deposits } & \multicolumn{4}{|c|}{ Time of scan (h) } & \multirow{2}{*}{$\underset{\text { CEA (mg/l) }}{\text { Serum }}$} & \multirow{2}{*}{$\begin{array}{l}\% \text { of iodine } \\
\text { excreted } \\
\text { in } 24 \mathrm{~h}\end{array}$} \\
\hline & Site & Identified & Site & Identified & $+\frac{1}{2}$ & +4 & +24 & +48 & & \\
\hline 1 & Colon & 0 & Liver & + & + & + & + & + & 210 & \\
\hline 2 & Rectum & 0 & Liver & + & + & - & - & $N$ & 16000 & \\
\hline 3 & Colon & 0 & Liver & - & - & - & - & $\mathrm{N}$ & 195 & \\
\hline 4 & Rectum & 0 & Liver & + & + & + & + & + & 410 & \\
\hline 5 & Rectum & 0 & Liver & + & - & I & + & - & 900 & 11 \\
\hline 6 & Colon & 0 & Liver & - & - & - & - & - & 225 & \\
\hline 7 & Rectum & 0 & Pelvis & + & - & + & + & + & 95 & 9 \\
\hline 8 & Colon & 0 & Liver & + & - & + & - & - & 750 & 14 \\
\hline 9 & Rectum & + & Peritoneum & + & + & + & + & + & 50 & \\
\hline 10 & Rectum & + & & 0 & - & + & + & - & 14 & \\
\hline 11 & Rectum & + & & 0 & - & - & - & + & 12 & 7 \\
\hline 12 & Pancreas. & + & Liver & + & + & + & + & $\stackrel{+}{-}$ & 300 & 14 \\
\hline 13 & Undetermined & - & $\begin{array}{l}\text { Peritoneum and } \\
\text { liver }\end{array}$ & - & $\mathrm{N}$ & - & $\mathrm{N}$ & $\mathrm{N}$ & 65 & \\
\hline 14 & & 0 & & $\overline{0}$ & - & - & N & $\vec{N}$ & $\begin{array}{l}03 \\
25\end{array}$ & \\
\hline 15 & & 0 & & 0 & - & - & - & $\bar{N}$ & 13 & 4 \\
\hline
\end{tabular}

$+=$ Tumour identified, $-=$ tumour not identified, $0=$ absent or resected, $\mathrm{N}=$ scan not performed.

widely disseminated, poorly differentiated adenocarcinoma but laparotomy failed to detect the primary tumour and it was thought unlikely to have been gastrointestinal. Furthermore, only one scan was performed (at four hours), and later scans, had they been carried out, might have been positive. In another patient with a false-negative scan computed tomography showed a huge solitary liver metastasis and the possibility arises that this was largely avascular. In addition, antibody preparation was less than satisfactory in this patient as radioactivity disappeared from the blood more rapidly than in the others. There seems to be no explanation for the failure of the method in case 6 .

Apart from these three patients, tumour localisation was possible in all patients, including one with carcinoma of the pancreas with secondary deposits (case 12). This primary tumour together with three others was clearly shown, and in one case (fig 1) local extension to the right pelvic wall was also seen. It is too early to know how precisely tumour masses can be identified but comparison with conventional colloidal technetium liver scans (figs 3 and 4 ) suggests that the image produced by anti-CEA antibodies is at least as satisfactory.

A major advantage of the method follows from the positive nature of the image. Thus tumour deposits can be identified at sites other than liver and colon-for example, lymph nodes and serous cavities.

Identification of deposits during follow-up after primary resection might enable assessment of other treatments-surgical, chemical, and radiation-at the intermediate stage of cancer development between localised and terminal disease.

Theoretically, there is more than enough CEA present in the serum fully to occupy the binding sites on the relatively small amount of antibody injected. It was therefore surprising to find a successful scan in a patient with a serum CEA as high as $16000 \mathrm{mg} / \mathrm{l}$. There was no consistent trend in the changes in serum CEA concentrations in our patients, with three showing no significant change and others showing falls of up to $50 \%$ which returned to preinjection levels over 24 hours. It is well established that CEA is heterogeneous ${ }^{12}$ and this might be the explanation for our finding. The CEA we have used for sheep immunisation has been prepared from hepatic metastases, so the antibody produced may have a higher affinity for tissue CEA than for serum CEA. This suggestion awaits further investigation.

The antiserum used was a sheep IgG carefully purified by immunoadsorption and affinity chromatography. Whether all steps are necessary to achieve tumour localisation has yet to be proved, and our later scans were carried out without using the immunoabsorbent column. Modification of the antiserum to improve specificity is possible, and if multiple injections are necessary then fragmentation of the protein to the Fab fragment might reduce reactions to later injections.

The use of an IgG preparation with purification by immunoabsorption to produce satisfactory tumour localisation in this pilot study is therefore encouraging, and there seems to be good potential for improving the antiserum further and obtaining fragments suitable for multiple injections. We hope that other and more tumour-specific antigens will be available for study, such as those derived from cancer of the breast ${ }^{13}$ and bronchus. ${ }^{14}$ Whether there is potential for carrying a therapeutic agent to the neoplasm depends on the degree of selective tumour localisation and the substance carried. It is, however, an encouraging possibility and one that needs further careful and extensive study.

We thank Professor A M Neville and Dr J Westwood, Institute of Cancer Research, Royal Cancer Hospital, who supplied purified CEA; Dr J McCartney, department of pathology, who monitored the adsorption of the antiserum by immunochemical staining; and $\mathrm{Dr} F$ Burrows, department of radiology, who helped with isotope and computed tomography scans. The work was supported by a grant from the Medical Research Council.

\section{References}

${ }_{1}^{1}$ Pressman D. Radiolabelled antibodies. Ann NY Acad Sci 1957;69:644-50.

2 Order SE. The history and progress of serologic immunotherapy and radiodiagnosis. Radiology 1976;118:219-23.

${ }^{3}$ Khoo SK, Warner NL, Lie JT, Mackay IR. Carcinoembryonic antigenic activity of tissue extracts : a quantitative study of malignant and benign neoplasms, cirrhotic liver, normal adult and fetal organs. Int $\mathfrak{f}$ Cancer 1973;11:681-7.

${ }^{4}$ Gold P, Krupey J, Ansari H. Position of the carcinoembryonic antigen of the human digestive system in ultrastructure of tumor cell surface. f Nat Cancer Inst 1970;45:219-25.

5 Von Kleist S, Burtin P. Localization cellulaire d'un antigene embryonnaire de tumeurs coliques humanes. Int f Cancer 1969;4:874-9.

${ }^{6}$ Primus FJ, MacDonald R, Goldenberg DM, Hansen HJ. Localization of GW39 human tumors in hamsters by affinity-purified antibody to carcinoembryonic antigen. Cancer Res 1977;37:1544-7.

7 Primus FJ, Wang RH, Goldenberg DM, Hansen HJ. Localization of human GW39 tumors in hamsters by radiolabelled heterospecific antibody to carcinoembryonic antigen. Cancer Res $1973 ; 33: 2977-82$.

${ }^{8}$ Goldenberg DM, Preston DF, Primus FJ, Hansen HJ. Photoscan localization of GW39 tumors in hamsters using radiolabelled anticarcinoembryonic antigen immunoglobulin G. Cancer Res $1974 ; 34: 1-9$.

${ }^{-}$Mach JP, Carrel S, Merenda C, Sordat B, Cerottini JC. In-vivo localization of radiolabelled antibodies to carcinoembryonic antigen in human colon carcinoma grafted into nude mice. Nature 1974 ;248:704-6.

${ }^{10}$ Reif AE, Curtis LE, Duffield R, Shauffer IA. Trial of radiolabelled antibody localization in metastases of a patient with tumor containing carcinoembryonic antigen (CEA). $\mathcal{f}$ Surg Oncol 1974;6:133-50.

11 Goldenberg DM, Deland F, Kim E, et al. Use of radiolabelled antibodies to carcinoembryonic antigen for the detection and localization of diverse cancers by external photoscanning. N Engl f Med 1978;298:1384-8.

12 Terry WD, Henkart PA, Coligan JE, Todd CW. Carcinoembryonic antigen: characterization and clinical applications. Transplant Rev 1974 ;20:100-29.

13 Woods KL, Cove DH, Morrison JM, Heath DA. The investigation of Lactalbumin as a possible marker for human breast cancer. Europ $\mathcal{F}$ Cancer 1979;15:47-51.

14 Gennings JN, Leake BA, Bagshawe KD. A human bronchogenic cancer antigen. In: Lehman FG, ed. Carcinoembryonic Proteins. Amsterdam: Elsevier, North Holland, 1979:553-8.

(Accepted 26 October 1979) 